\title{
Approximating heat resistance of nickel- based superalloys by a sigmoid
}

Cite as: AIP Conference Proceedings 2293, 140019 (2020); https://doi.org/10.1063/5.0026744

Published Online: 25 November 2020

Dmitry A. Tarasov, Andrey G. Tyagunov, and Oleg B. Milder

\section{ARTICLES YOU MAY BE INTERESTED IN}

Modeling the heat resistance of nickel-based superalloys by a deep learning neural network AIP Conference Proceedings 2293, 140020 (2020); https://doi.org/10.1063/5.0026745

Gradation trajectories of ideal initial printing colorants in electrophotography: Discrete computation

AIP Conference Proceedings 2293, 140016 (2020); https://doi.org/10.1063/5.0026742

The inverse problem of spectral reflection prediction: Problems of framework selection AIP Conference Proceedings 2293, 140012 (2020); https://doi.org/10.1063/5.0026741

Challenge us.

What are your needs for periodic signal detection?

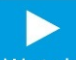

Watch

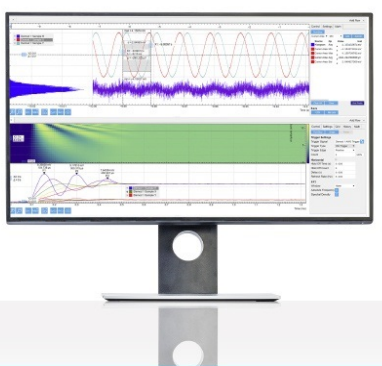

framework selection
$10.1063 / 5.0026741$ 


\title{
Approximating Heat Resistance of Nickel-based Superalloys by a Sigmoid
}

\author{
Dmitry A. Tarasov ${ }^{1, a)}$, Andrey G. Tyagunov ${ }^{1, b)}$, Oleg B. Milder ${ }^{1, c)}$ \\ ${ }^{1}$ Ural Federal University, Mira str., 19, Ekaterinburg, RUSSIA 620002 \\ a)Corresponding author: datarasov@yandex.ru \\ b)adi8@yandex.ru \\ c)milder@mail.ru
}

\begin{abstract}
The nickel-based superalloys are unique materials with complex doping applied to manufacturing the gas turbine engine parts. The alloys show resistance to mechanical and chemical degradation under high pressure, high temperature, and long-term isothermal exposures. One of the main alloys' service properties is the heat resistance. Numerically, it is expressed in the tensile strength values (MPa). Simulation of the heat resistance behavior is an important engineering task, which would significantly simplify the analysis of existing and designing the new alloys. In this paper, we use results of the heat resistance simulation by an artificial neural network, as well as, experimental data for approximating the changes in the heat resistance $v s$ isothermal exposures expressed in the complex Larson-Miller parameter by a sigmoidal function.
\end{abstract}

Keywords: Nickel-based superalloys, Sigmoid, Simulation, Tensile strength, Larson-Miller parameter.

\section{INTRODUCTION}

The nickel-based superalloys are used to manufacture the most important heat resisting parts of gas turbine engines. The basis of the alloys is nickel as the isomorphic material. The rest content consists of significant amount of other doping [1-13]. The major service properties of the alloys are the heat resistance and structural thermal stability. The heat resistance is the ability of a material to resist the load at elevated temperatures without undergoing permanent deformation or fracture. To quantify the heat resistance, mechanical tests on long-term tensile strength $(\sigma$, $\mathrm{MPa}$ ) are carried out. This variable shows the greatest mechanical stress that the material could resist without breaking at a given temperature, exposure time, and working conditions. The magnitude of the tensile strength is denoted as $\sigma_{t}^{\tau}$, where $\tau$ is the isothermal holding time in hours, and $t$ is its temperature in ${ }^{\circ} \mathrm{C}$. The thermal stability is the ability of a material to retain properties during a period of the prolonged isothermal exposures [14].

Up-to-date, several hundred compositions of the high-temperature alloys are known and still being developed. The current level of the heat resistance is achieved due to optimization of the chemical composition, as well as, the application of the most advanced technology of single crystal casting. However, the desire to increase the characteristics of the gas turbines has led to a significant increase in their cost, especially, due to alloying with expensive elements. Hence, new alloys development is a substantial engineering problem, one of the key issue of which is a low alloy cost and ease of production while maintaining the desired performance.

Since the physical experiments under the alloys are time consuming and quite expensive, the mathematical modeling might be a method that would ease the design works. The problem is the fact that the alloys are tested in fairly narrow time and temperature ranges. Thus, the database on alloys service properties for modeling is insufficient for building the full-fledged statistical models. Nevertheless, novel computational methods make it possible to extrapolate the results of tests for the long-term strengths without the implementation of expensive and long-term full-scale experiments. The first method that comes to mind is a neural network approach. The artificial neural networks (ANN) are ones of the most powerful and modern modeling techniques based on a statistical

International Conference of Numerical Analysis and Applied Mathematics ICNAAM 2019

AIP Conf. Proc. 2293, 140019-1-140019-4; https://doi.org/10.1063/5.0026744 Published by AIP Publishing. 978-0-7354-4025-8/\$30.00 
approach close to practical application. The use of neural networks is suitable for modeling correlations that are difficult to describe or which cannot be accurately predicted by the physical models. Since artificial modeling of the neural networks is a non-linear statistical method, it can be used to solve problems that are not amenable to solution by the conventional statistical methods [15].

Previously, the artificial neural networks were used to analyze the nickel-based alloys [16, 17], but the goal of these works was to synthesize new chemical compositions of the heat-resistant alloys [16, 18-21], to model change in the coefficient of thermal expansion [22, 23], to model energy hysteresis [24], to predict a low-cycle fatigue energy [25], to model the development of fatigue cracks [26], to predict the occurrence of material defects [27], to model the time to failure [28]. Moreover, it has done mainly about the same grade of the alloy.

It did not succeeded to find works devoted to calculation of the heat resistance on the basis of establishing the following important relation. Namely, one between the alloy chemical composition and the experimental values of the long-term tensile strength. Also, there are no works with analysis of the changes in the heat resistance and thermal stability of a whole number of alloys.

Methods for comparing the results of testing the samples that have undergone various isothermal exposures are based on the introduction of a parameter that reflects the simultaneous influence of both temperature and time. It is the Larson-Miller parametric coordinates $\left(P_{L M}\right)$. According to this technique, the long-term strength limit $\sigma_{t}^{\tau}$ is represented as a function of the Larson-Miller parameter $\sigma_{t}^{\tau}=f\left(P_{L M}\right)$. For the heat-resistant nickel alloys, $P_{L M}$ has the form $P_{L M}=T(20+\lg \tau)$.

The Bayesian regularized artificial neural network (BRANN) is more robust than the standard back-propagation ones and might reduce or even eliminate the need for cross-validation. It has shown its satisfactory predictive ability along with the resistance to overtraining during application in the similar tasks [29]. Based on that, we have previously predicted service properties of the superalloys accounting their chemical composition [30]. However, not all chemical elements have the same effect on the properties of alloys.

The alloys database collected by authors contains the test information and service properties on 308 nickel-based superalloys. In total, 47 pairs $(\tau, t)$ were taken into account. The samples include 23 alloying elements: $\mathrm{C}, \mathrm{Cr}, \mathrm{Co}$, Mo, W, Al, Ti, Nb, B, Fe, Y, Zr, Ta, Re, Ru, V, Ce, La, Si, Mn, Hf etc. (Ni is balanced). For each alloy in the database, no more than 25 test results $\left(\sigma_{t}^{\tau}\right)$ were known. Each alloy forms as many samples for the network training as many different physical tests were conducted. The main task of the preliminary computational experiment was to fill in the missing $\sigma_{t}^{\tau}$ values in the database and to evaluate the prediction ability of the model. To create and train the networks, the MATLAB software was applied. The input parameters of the models are the chemical composition of the alloys and service properties.

In this work, using some common alloys samples from the same database [30], we take a step towards the analytical approximation of the $\sigma_{t}^{\tau}$ vs $P_{L M}$ dependence and use the sigmoidal function for this purpose.

\section{APPROACH AND EXPERIMENTAL}

For verification of the simulation result, we extracted 9 randomly selected common superalloys from the database before the training. This sub-sample served for the model performance evaluation by calculating the error between the real and predicted values. The chemical compositions of the alloys are shown in Table 1.

TABLE 1. Chemical compositions of the verification sub-sample, wt $\%$ ( $\mathrm{Ni}$ is balansed).

\begin{tabular}{|c|c|c|c|c|c|c|c|c|c|c|c|c|c|c|}
\hline Alloys & $\mathrm{Cr}$ & Co & Mo & $\mathbf{W}$ & Al & $\mathbf{T i}$ & Ta & Nb & $\operatorname{Re}$ & $\mathbf{R u}$ & Hf & C & B & $\mathbf{Z r}$ \\
\hline AM1 & 7.0 & 8.0 & 2.0 & 5.0 & 5.0 & 1.8 & 8.0 & 1.0 & - & - & - & - & - & - \\
\hline AM3 & 8.0 & 5.5 & 2.25 & 5.0 & 6.0 & 2.0 & 3.5 & - & - & - & - & - & - & - \\
\hline CM186LC & 6.0 & 9.3 & 0.5 & 8.4 & 5.7 & 0.7 & 3.4 & - & 3.0 & - & 1.4 & 0.07 & 0.015 & 0.005 \\
\hline CM247LC & 8.0 & 9.3 & 0.5 & 9.5 & 5.6 & 0.7 & 3.2 & - & - & - & 1.4 & 0.07 & 0.015 & 0.010 \\
\hline CMSX-2 & 8.0 & 5.0 & 0.6 & 8.0 & 5.6 & 1.0 & 6.0 & - & - & - & - & - & - & - \\
\hline CMSX-3 & 8.0 & 4.8 & 0.6 & 8.0 & 5.6 & 1.0 & 6.3 & - & - & - & 0.1 & - & - & - \\
\hline CMSX-4 & 6.5 & 9.6 & 0.6 & 6.4 & 5.6 & 1.0 & 6.5 & - & 3.0 & - & 0.1 & - & - & - \\
\hline CMSX-6 & 10.0 & 5.0 & 3.0 & - & 4.8 & 4.7 & 6.0 & - & - & - & 0.1 & - & - & - \\
\hline CMSX-10 & 2.0 & 3.0 & 0.4 & 5.0 & 5.7 & 0.2 & 8.0 & - & 6.0 & - & 0.03 & - & - & - \\
\hline
\end{tabular}


Since the structure of superalloys is heterophasic, the process of its degradation is multi-stage due to the different temperature stability of the structural components. For most alloys, the dependence $\sigma\left(P_{L M}\right)$ demonstrates a noticeable nonlinearity and the asymptotic tendency of $\sigma$ to zero with $P_{L M}$ greater than 30. On the other hand, at relatively low temperatures and short times $\left(P_{L M}<20\right), \sigma$ decreases slightly and remains almost constant to a certain critical value of the $P_{L M}$. In the $P_{L M}$ range from 20 to 30, all alloys have a practically linear $\sigma$ decrease. We believe that this behavior can be described in the most optimal way by the logistical function of the following form under the assumption that $x \equiv P_{L M}$ :

$$
\sigma(x)=\sigma_{2}+\frac{\sigma_{1}-\sigma_{2}}{1+\exp \left(\frac{x-x_{0}}{p}\right)}
$$

were $\sigma_{1}, \sigma_{2}, x_{0}, p$ are the parameters that are established during the approximation; $\sigma_{2}$ is the level of the asymptotic tendency of $\sigma$ to zero with $P_{L M}$ greater than 30; $\sigma_{1}$ is the level of almost constant $\sigma$ behavior with $P_{L M}$ lower than 20; and $x_{0}$ is the inflection point geometrically corresponding to the middle of the range of the linear reduction of $\sigma$. The most physically inspired parameter in model (1) is the slope factor $p$. This parameter is responsible for the description of the thermo-temporal dynamics of the alloys phase stability. The higher the value of $p$, the slower the process of the thermo-temporary degradation of the alloy structure goes.

\section{RESULTS AND DISCUSSION}

The dependencies of the tensile strength $v_{S} P_{L M}$ have been built for some common superalloys that were collected in the sub-sample (Table 1). The plots (Fig. 1) show the model predictions of the tensile strengths together with the real experimental data and with approximations by relation (1). The figures show the satisfactory accuracy of the both curves: ANN predictions and the sigmoidal approximation. The introduced slope factor $p$ characterizes how close the curve lays to the abscissa axis. So, we may deduce that $p$ is an indirect indicator of the alloy thermal stability.
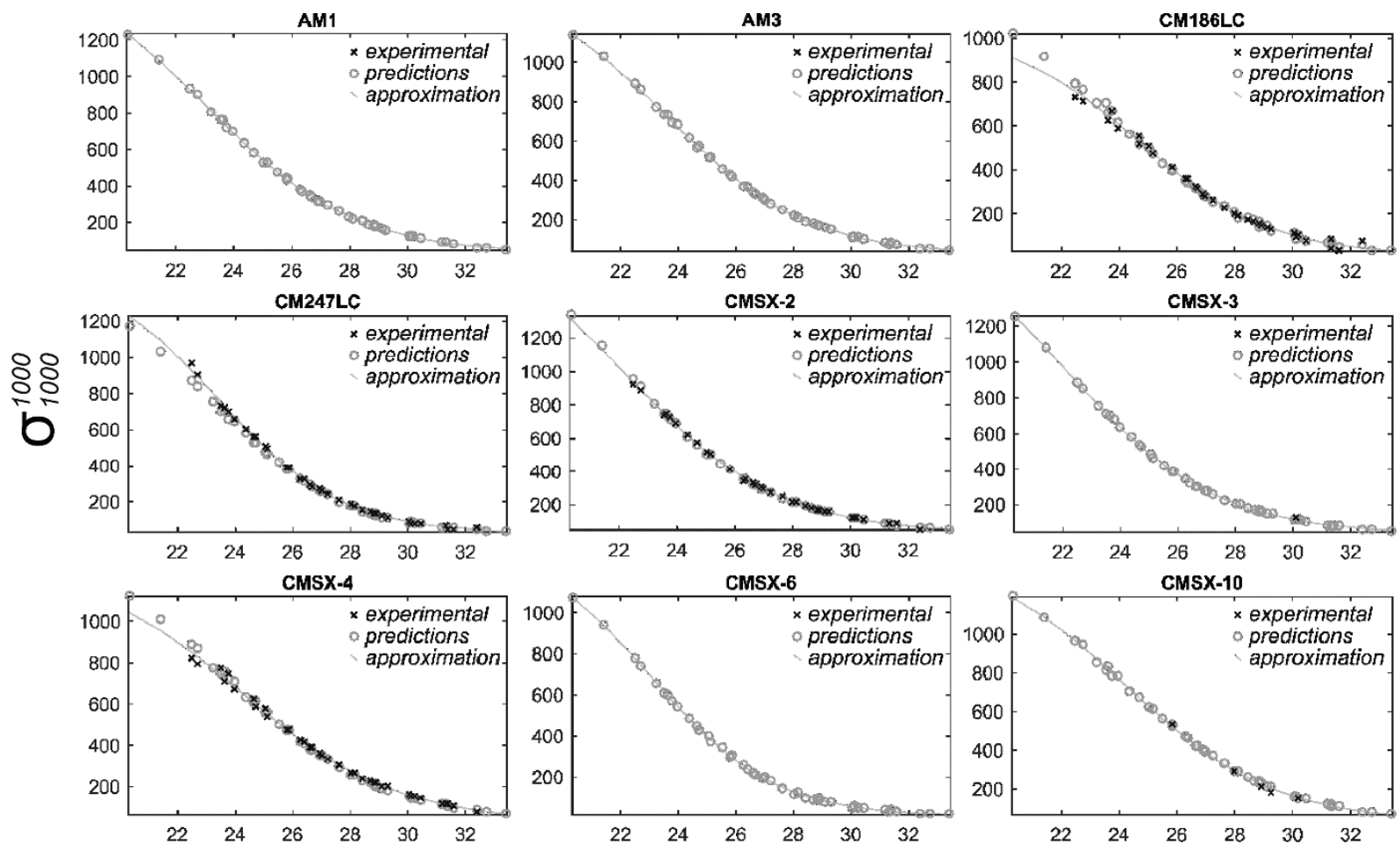

Larson-MIller parameter

FIGURE 1. Results of prediction and approximation for particular alloys from the verification sub-sample. 
The mean squared errors between real data, predictions, and approximations were calculated. For more than $95 \%$ of the dataset, the prediction error is located within $\pm 5 \%$. The RRMSE does not exceed 0.20 for most observations, moreover, the median value is 0.06 and $75 \%$ percentile is just 0.10 . Thus, the model might be considered practically valuable.

\section{CONCLUSION}

In our previous work, the model based on the Bayesian regulated artificial neural networks is applied to predict the missing service properties of the high-temperature nickel-based superalloys over the entire range of temperatures and exposures. Here, the known data on the characteristics of alloys with different chemical compositions were used. For modeling, a database of exposure conditions and service properties of 308 superalloys was collected and compiled by authors. To compare the heat resistance $\left(\sigma_{t}^{\tau}\right)$ of the alloys, an approximation using the complex LarsonMiller parameter is applied. The method allowed one to obtain missing data for all types of tests in the range of variation of the Larson-Miller parameter from 22 to 35.

Based on the predictions, we have built an analytical approximation model using a common logistical sigmoid. The approximation has shown a good prediction accuracy (about 5\% together with the ANN modeling). Hence, we may argue that the first analytical approximation created by the authors is useful and might be applied to modeling the behavior of the nickel-based superalloys.

\section{REFERENCES}

1. S.T. Kishkin and E.N. Kablov "Casting heat-resistant alloys for turbine blades" in Aviation materials. fav. tr. viam (anniversary collection). MISiS, VIAM (2002), pp. 48-58. (In Russian).

2. S. Walston, A. Cetel, R. Mac Kay, K. O'Hara, et al. "Joint development of a fourth generation single crystal superalloy" in Supralloy ed. K.A. Green (Publ. Minerals, Metals \& Materials Society. Seven springs Mountain Resort, Pen, 2004), pp. 15-24.

3. V.P. Kuznetsov, V.P. Lesnikov, and N.A. Popov, "Structure and properties of single-crystal high-temperature nickel alloy", UrFU, 2004, 160p. (In Russian).

4. S.N. Fedoseev "The composition and structure of heat-resistant alloy PWA 1480" in Proceedings of the XIX international scientific and practical conference of students, postgraduates and young scientists, Tomsk, (2013) pp. 174-175. (In Russian).

5. Y. Koizumi, T. Kobayashi, T. Yokokawa, Z. Jianxin, et al. "Development of next-generation Ni-based single crystal superalloys" in Supralloy ed. K.A. Green (Publ. Minerals, Metals \& Materials Society. Seven springs Mountain Resort, Pen, 2004), pp. 35-43.

6. A.G. Tiagunov, E.E. Baryshev, N.N. Stepanova "Influence of melt structure on the properties of Nickel based superalloys in the solid state", UrO RAS (2010) 198p. (In Russian).

7. A. Sato, H. Harada, A. Yeh, K. Kawagishi, et al. "A 5th generation of superalloys with balanced high temperature properties and processability" in Superalloys (2008), pp. 131-138.

8. High-temperature intermetallic alloys. https://viam.ru/review/2942 (in Russian).

9. N.V. Petrushin, I.L. Svetlov, O.G. Ospennikova "Cast nickel based superalloys" in All material. Encyclopedic reference book.№6 (2012) (In Russian).

10. E.N. Kablov, N.G. Nuts, V.N. Toloraya, I.M. Demonise "Casting heat-resistant Nickel alloys and technology for producing single-crystal turbine blades GTE in Light alloy technology, 4 (2002). (in Russian).

11. E.N. Kablov, V.N. Toloraya, N.G. Orehov "Single-crystal rhenium-containing Nickel alloys for turbine blades of GTE" in Science of metals and heat treatment of metals, 7 (2002). (In Russian).

12. S.V. Gaiduk "Design foundry high-temperature corrosion resistant nickel alloy for the manufacture of turbine blades by the method of directed (mono) crystallization" in Structural and functional materials. New materials and technologies in metallurgy and mechanical engineering, 1 (2016) (in Russian).

13. E.B. Argimbaeva, O.A. Bazyleva, E.I. Turenko "Intermetallic alloys based on Ni3Al" in All material. Encyclopedic reference book 5, (2012) (in Russian).

14. R.C. Reed "The Superalloys. Fundamentals and Applicatios", Cambridge University Press (2006), 372p.

15. S.O. Haykin "Neural Networks and Learning Machines", 3rd ed,, McMaster University, Ontario Canada (2009). 906p. 